Gynecologic and

Obstetric Investigation

\title{
A Comparison of Hemostatic Properties between Monopolar and Bipolar Hysteroscopic Surgery using Rotational Thromboelastometry: A Randomized Trial
}

\author{
Hyun-Jung Shin ${ }^{a}$ Hyo-Seok Na ${ }^{a}$ Ji-Yun Han ${ }^{a}$ Jung-Won Hwang ${ }^{a}$, b \\ ${ }^{a}$ Department of Anesthesiology and Pain Medicine, Seoul National University Bundang Hospital, \\ Seongnam-si, South Korea; ${ }^{b}$ College of Medicine, Seoul National University, Seoul, South Korea
}

\section{Keywords}

Hysteroscopic surgery - Blood coagulation .

Thromboelastometry · Absorption · Irrigation fluid

\begin{abstract}
Objective: Systemic absorption of irrigation fluid can lead to a disturbed coagulation cascade. We compared the changes in hemostatic properties on using nonelectrolyte solution in monopolar hysteroscopic surgery (HS) with that on using isotonic saline in bipolar HS via rotational thromboelastometry (ROTEM) analysis. Methods: A total of 54 patients were randomized into 2 groups: 1 group underwent monopolar HS using a mixture of $2.7 \%$ sorbitol and $0.54 \%$ mannitol (the $\mathrm{HS}_{\mathrm{MP}}$ group), whereas the other group underwent bipolar $\mathrm{HS}$ using $0.9 \%$ isotonic saline (the $\mathrm{HS}_{\mathrm{BP}}$ group). The effects of these 2 methods on coagulation were assessed via pre and postoperative laboratory tests, including estimation of the international normalized ratio of prothrombin time and activated partial thromboplastin time and ROTEM analysis. In addition, the hemoglobin, hematocrit, and electrolyte levels and the platelet count were analyzed. Results: Patient characteristics, volume of irrigation fluid absorbed, and type of procedure were comparable between the 2 groups. There were no significant differences in the pre and postoperative
\end{abstract}

values of ROTEM parameters between the 2 groups. Most postoperative ROTEM parameters, as compared with preoperative values, changed in both groups; clot formation time was prolonged, and the $\mathrm{a}$-angle and maximum clot firmness were decreased. All ROTEM parameters were maintained within the normal range. Hematological parameters, including hemoglobin and hematocrit levels and platelet count, were significantly decreased postoperatively in both groups compared to the preoperative values. No pre and postoperative hematological and hemostatic parameters were significantly different between the 2 groups. Conclusion: Irrigation fluid absorbed in healthy women during HS caused hypocoagulable changes in the blood, irrespective of the irrigant type, and no significant differences between HS using monopolar and bipolar electrodes were demonstrated.

(c) 2019 S. Karger AG, Basel

\section{Introduction}

While irrigation, fluid is widely used in various endoscopic surgeries, systemic absorption of irrigation fluid can cause various complications, such as electrolyte imbalance, mental confusion, brain edema, and even death $[1,2]$. Hysteroscopic surgery (HS) has been considered an

\section{KARGER}

(c) 2019 S. Karger AG, Basel

E-Mail karger@karger.com

www.karger.com/goi
Jung-Won Hwang

Department of Anesthesiology and Pain Medicine, Seoul National University Bundang Hospital, College of Medicine, Seoul National University

173-82 Gumiro, Bundang, Seongnam-si, Gyeonggi-do 13620 (South Korea)

E-Mail jungwon@snubh.org 
effective surgical intervention for the management of various gynecological conditions. Despite its popularity, HS has also been shown to be associated with the risk of irrigation fluid absorption.

Recently, a clinical investigation reported on the effect of electrolyte-free hypotonic irrigation solution on blood coagulation in patients undergoing transurethral prostatectomy using a monopolar electrode [3]. The average volume of irrigation fluid absorption has been known to be $400-700 \mathrm{~mL}$ during HS, which is slightly greater than that during transurethral prostatectomy $[1,4]$. Although the risk of bleeding is relatively low, perioperative bleeding remains a potential complication of HS [5, 6]. Moreover, coagulopathy can also be induced by irrigation fluid absorption $[7,8]$. Nevertheless, to the best of our knowledge, no previous study has evaluated the effect of irrigation fluid on blood coagulation using point-of-care hemostasis monitoring, such as rotational thromboelastometry (ROTEM), in HS.

Hence, the purpose of this study was to evaluate the effect of irrigation fluid on blood coagulation in patients undergoing HS and to identify differences between the use of a nonelectrolyte solution in monopolar HS and isotonic saline in bipolar HS. On the basis of the hypothesis that absorption of irrigation fluids causes changes in the coagulation cascade, we performed the present randomized study using ROTEM analysis.

\section{Materials and Methods}

\section{Ethical Approval}

After obtaining approval from the Institutional Review Board of Seoul National University Bundang Hospital (Seongnam-si, South Korea, approval on November 26, 2014, B-1411/274-004), this study was registered in the Clinical Research Information Service (http://cris.nih.go.kr, KCT0001330). Written informed consent was obtained from all participating subjects.

\section{Study Population}

This randomized study was performed in 54 female patients with an American Society of Anesthesiologists (ASA) physical status of 1 or 2 who were scheduled to undergo HS for the resection of polyps or fibroids under general anesthesia. Preoperative exclusion criteria included medication interfering with hemostasis (e.g., aspirin, clopidogrel, or warfarin), hematological disease, pulmonary diseases such as pulmonary edema or effusion, renal disorders, and hepatic disorders.

\section{Intervention}

These 54 patients were randomized into 2 groups: 1 group underwent monopolar HS, using a mixture of $2.7 \%$ sorbitol and 0.54\% mannitol (Urosol ${ }^{\mathrm{TM}}$, CJ Pharma., Seoul, Korea; group $\mathrm{HS}_{\mathrm{MP}}$ ), whereas the second group underwent bipolar HS, using
0.9\% isotonic saline (CJ Pharma., Seoul, Korea; group $\mathrm{HS}_{\mathrm{BP}}$ ). The randomization chart was generated using a web-based system (www.randomization.com) that utilizes the Wichmann and Hill number generator as modified by McLeod. The allocation ratio was 1:1. One investigator, who was not directly involved in the anesthetic management of the patients, generated the random allocation sequence and assigned participants to interventions.

\section{Anesthesia and Operation}

All patients were premedicated with midazolam. Standard monitoring (pulse oximetry, electrocardiogram, and blood pressure) was applied, and the bispectral index (BIS ${ }^{\mathrm{TM}}$, Covidien Icn., USA) was monitored to maintain suitable anesthetic depth (40$60)$. General anesthesia was induced and maintained using propofol (Fresofol ${ }^{\circledR}$, Fresenius Kabi, Korea Ltd., Korea) and remifentanil (Ultiva ${ }^{\circledR}$, GlaxoSmithKlein, UK) with a target-controlled infusion device (Orchestra ${ }^{\circledR}$, Fresenius vial, France). Laryngeal mask airway (i-gel ${ }^{\circledR}$, Intersurgical Ltd., UK) was inserted for ventilation support. Ringer's lactate solution was infused at a rate of $5 \mathrm{~mL} /$ $\mathrm{kg} / \mathrm{h}$. The hysteroscopic operation was conducted by 3 operators.

Irrigation fluid was flushed at a flow rate of $200 \mathrm{~mL} / \mathrm{min}$ and a continuous pressure of $70 \mathrm{~mm} \mathrm{Hg}$. The amount of irrigation fluid absorbed was recorded using the volumetric fluid balance method. Blood loss influences the accuracy of fluid balance, and given that there is generally less blood loss in endometrial resection than in transurethral prostatectomy, use of the volumetric fluid balance is appropriate for endometrial resection [1]. The irrigation fluid was collected by passive drainage into a closed plastic bottle. In addition, the amount of irrigation fluid, which flowed into the bag in the surgical field, was recorded. The amount of absorbed fluid was calculated as the difference between the infused and collected irrigation fluid.

In our institution, irrigation fluid balance is checked every 30 min during HS to avoid intravasation syndrome. When the amount of irrigation fluid absorbed is $>1,000 \mathrm{~mL}$ or the operation time exceeds $1 \mathrm{~h}$, laboratory tests, including assessment of hematological parameters and electrolyte levels and blood gas analysis, were performed to monitor the excessive absorption of irrigation fluid using a point-of-care device.

\section{Hematological Variables and ROTEM Analysis}

Using the 2 -syringe technique to avoid contamination by tissue factors, we collected venous blood samples from patients twice, once preoperatively and once postoperatively (upon arrival at the postanesthetic care unit). After discarding the initial blood sample (about $5 \mathrm{~mL}$ ), the test blood sample was taken immediately. The obtained blood samples were put into EDTA-containing bottles (Becton Dickinson, Plymouth, UK) to analyze the hemoglobin and hematocrit levels and platelet count and also into serum separator bottles to determine the electrolytes levels (sodium, potassium, and chloride). To assess the international normalized ratio of prothrombin time and activated partial thromboplastin time and to perform ROTEM analysis (Pentapharm, Munich, Germany), blood samples were collected in citrate-containing bottles.

ROTEM analyses were performed by 1 blinded investigator who was not directly involved in the anesthetic care of the patients, according to the manufacturer's instructions. The following 4 ROTEM parameters were obtained: clotting time (CT), $\alpha$-angle (a), clot formation time (CFT), and maximum clot firmness (MCF). EXTEM and INTEM tests, which indicate extrinsic and 
Fig. 1. CONSORT flow chart. ROTEM, rotational thromboelastometry; HS, hysteroscopic surgery.

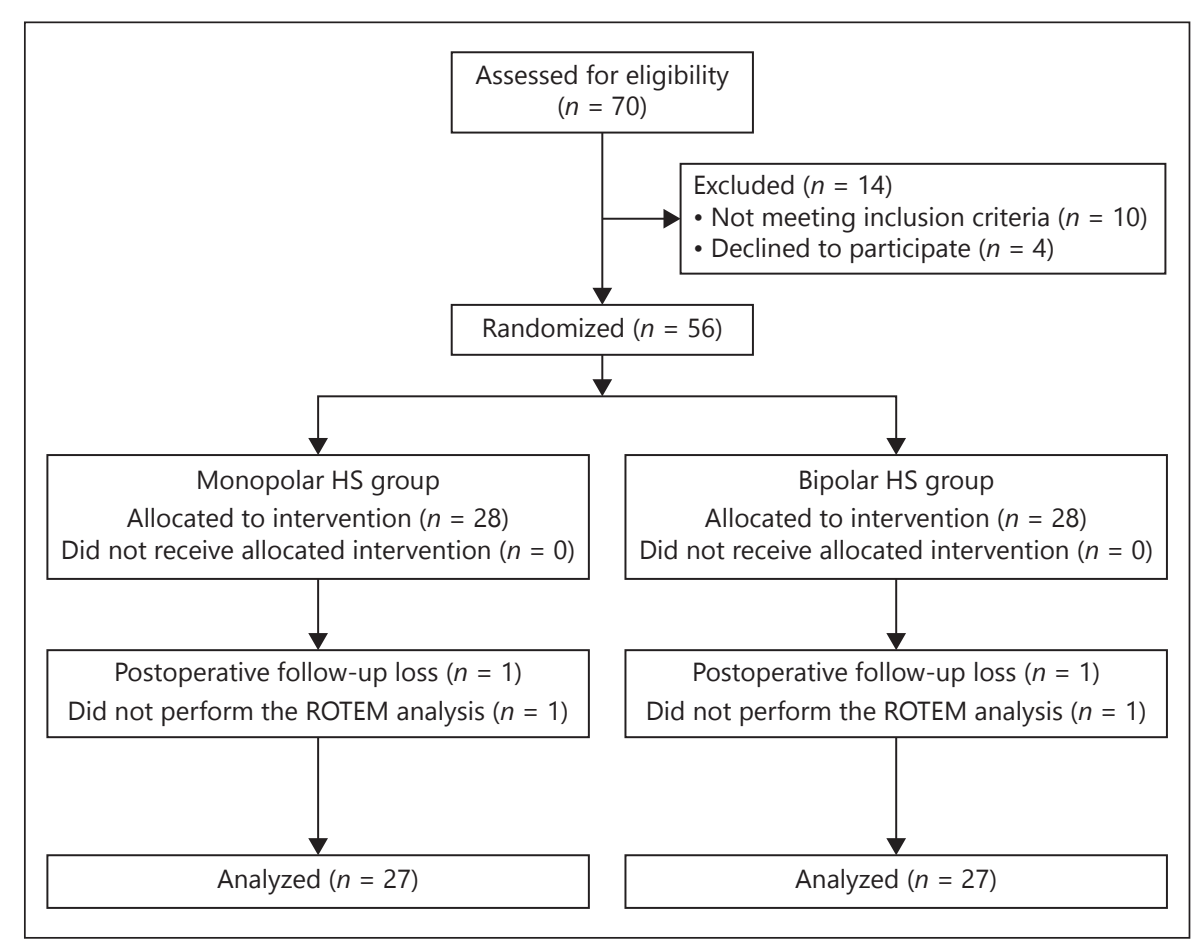

intrinsic coagulation pathways, respectively, were performed using test reagents (star-TEM ${ }^{\circledR}$ with ex-TEM ${ }^{\circledR}$ or in-TEM ${ }^{\circledR}$ ). Changes in the fibrinolytic system, including fibrinogen concentration and fibrin polymerization, were examined by the FIBTEM test using fib-TEM ${ }^{\circledR}$ and ex-TEM ${ }^{\circledR}$ reagents. Recommended reagents were mixed with $300 \mu \mathrm{L}$ of citrated whole blood for each test by computer-assisted pipetting. These ROTEM $^{\circledR}$ tests were performed within $10 \mathrm{~min}$ after collecting the blood samples.

Estimated blood loss was calculated using the formulas previously described by Rosencher et al. [9] with the pre and postoperative hematocrit values.

\section{Sample Size}

The primary outcome was the MCF of FIBTEM. Secondary outcomes were the results of ROTEM (INTEM and FIBTEM variables), hemoglobin, hematocrit, platelet count, international normalized ratio of prothrombin time, activated partial thromboplastin time, electrolytes (sodium, potassium, and chloride), volume of irrigation fluid absorbed, anesthesia time, operating time, and total amount of irrigation fluid. The pilot data (MCF of FIBTEM differences between pre and postoperative values, mean [SD]) from 30 patients were as follows: $\mathrm{HS}_{\mathrm{MP}}$ group MCF of FIBTEM value difference, 2.4 (1.2); $\mathrm{HS}_{\mathrm{BP}}$ group MCF of FIBTEM value difference, 1.8 (1.0). Assuming this difference and aiming for a power of $80 \%$ and a risk of 0.05 for a type 1 error, 25 patients were required for each group. We selected 28 patients per group, anticipating a $10 \%$ dropout rate.

\section{Statistical Analysis}

Data are expressed as median (interquartile range) or number (percentage). All variables were tested for normality using the Shapiro-Wilk test. Statistical analyses were performed using the
Mann-Whitney U test for continuous data and the chi-square test for categorical data. Data were analyzed using SPSS version 22 (SPSS Inc., Chicago, IL, USA) software. A $p$ value of $<0.05$ was considered statistically significant.

\section{Results}

Patients were included between January 2015 and June 2017. Of a total of 70 patients who were evaluated for eligibility, 56 were assigned to one of the 2 groups. Two patients who did not undergo postoperative ROTEM analysis were excluded during the follow-up period. Otherwise, no other patients were excluded from the analysis (Fig. 1). No complications such as hyponatremia or massive bleeding, occurred during the surgery. Patient characteristics and surgical and anesthetic data are shown in Table 1.

Data on the ROTEM parameters are described in $\mathrm{Ta}$ ble 2. There were no significant differences in the pre and postoperative values of the ROTEM parameters between the 2 groups. The postoperative ROTEM parameters, as compared with the preoperative values, changed in both groups toward a hypocoagulable pattern in which the CFT was prolonged and the $a$-angle and MCF were decreased. In the $\mathrm{HS}_{\mathrm{MP}}$ group, postoperatively, the CFT of INTEM was significantly prolonged $(p=0.028)$ and the MCF of INTEM was significantly decreased $(p=0.021)$. 
Table 1. Patients, surgery, and anesthesia characteristics

\begin{tabular}{|c|c|c|c|}
\hline & $\mathrm{HS}_{\mathrm{MP}}(n=27)$ & $\mathrm{HS}_{\mathrm{BP}}(n=27)$ & $p$ value \\
\hline Age, years & $49(44-57)$ & $48(46-59)$ & 1.000 \\
\hline Weight, kg & $54(52-65)$ & $53(49-62)$ & 0.071 \\
\hline Height, m & $158(156-162)$ & $157(153-160)$ & 0.155 \\
\hline BMI, $\mathrm{kg} / \mathrm{m}^{2}$ & $22(21-24)$ & $21(20-24)$ & 0.185 \\
\hline Surgery time, $\min$ & $25(15-35)$ & $20(15-30)$ & 0.267 \\
\hline Anesthesia time, min & $50(45-60)$ & $50(45-60)$ & 0.771 \\
\hline Crystalloid, mL & $200(200-350)$ & $250(200-300)$ & 0.958 \\
\hline Amount of irrigation, $\mathrm{mL}$ & $1,500(1,000-3,300)$ & $1,800(1,200-3,000)$ & 0.684 \\
\hline Amount of absorption, $\mathrm{mL}$ & $200(100-200)$ & $200(100-200)$ & 0.504 \\
\hline Estimated blood loss, $\mathrm{mL}$ & $114(0-228)$ & $94(0-202)$ & 0.274 \\
\hline Preoperative diagnosis & & & 0.413 \\
\hline Polyp & $14(52)$ & $11(41)$ & \\
\hline Leiomyoma & $13(48)$ & $16(59)$ & \\
\hline Pathologic findings & & & 0.675 \\
\hline Polyp & $13(48)$ & $11(41)$ & \\
\hline Leiomyoma & $11(41)$ & $13(48)$ & \\
\hline Polyp + leiomyoma & $2(7)$ & $3(11)$ & \\
\hline Endometrial hyperplasia & $1(4)$ & $0(0)$ & \\
\hline
\end{tabular}

Moreover, the postoperative CT $(p=0.004)$ and CFT $(p=$ 0.001) of EXTEM were increased significantly. The MCF of FIBTEM showed a significant decrease postoperatively $(p=0.001)$. In the $\mathrm{HS}_{\mathrm{BP}}$ group, postoperatively, the CFT of INTEM was significantly increased $(p<0.001)$, and the $\alpha$-angle $(p=0.001)$ and MCF $(p=0.043)$ of INTEM were significantly decreased. Moreover, the postoperative CFT of EXTEM was prolonged significantly $(p=0.001)$, and the postoperative $\alpha$-angle $(p=0.009)$ and $\operatorname{MCF}(p=0.014)$ of EXTEM were decreased significantly. In addition, the MCF of FIBTEM was decreased postoperatively ( $p=$ $0.005)$. However, there was no statistically significant difference between the preoperative and postoperative values with respect to the CT and $\alpha$-angle of INTEM and the MCF of EXTEM in the $\mathrm{HS}_{\mathrm{MP}}$ group; likewise, there were no significant difference between the preoperative and postoperative values with respect to the CT of INTEM and EXTEM in the $\mathrm{HS}_{\mathrm{BP}}$ group. All ROTEM values were maintained within the normal range.

Hematological parameters, including hemoglobin, hematocrit, and platelet count, were significantly decreased postoperatively in both the $\mathrm{HS}_{\mathrm{MP}}$ and $\mathrm{HS}_{\mathrm{BP}}$ groups compared with the preoperative values (Table $3, p<0.001$ ). None of the hematological and hemostatic parameters or the electrolytes were significantly different between the 2 groups pre and postoperatively (Table 3 ).

Hemostasis and Irrigation Fluid in Hysteroscopic Surgery

\section{Discussion}

The present study focused on the effect of absorbed irrigation fluid on hemostatic properties. Interestingly, both the electrolyte-free hypotonic and isotonic irrigation solutions shifted the coagulation cascade toward a hypocoagulable state (prolonged CFT, decreased $\alpha$-angle, and MCF), showing no differences in the ROTEM values between the 2 fluids. In addition, all ROTEM parameters were within the reference range.

Traditionally, nonelectrolyte hypotonic solutions containing a mixture of mannitol and sorbitol or glycine have been used as irrigation fluids, allowing monopolar HS to be used for coagulation and tissue resection $[4,10]$. However, these low-viscosity distending media carry a substantial risk of rapid fluid absorption that may result in fluid overload, dilutional hyponatremia, and a variety of concomitant side effects, including coagulopathy $[2,5,7]$. With recent technological advancements, HS using a bipolar electrical system, with isotonic saline as the distending medium, has been developed [11]. Although bipolar HS may mitigate the risk of dilutional hyponatremia, which can be caused by nonelectrolyte hypotonic irrigation solutions, the risk of intravasation of irrigation fluid remains and may lead to hyperchloremic acidosis or dilutional coagulopathy [8]. 
Table 2. ROTEM parameters

\begin{tabular}{|c|c|c|c|c|c|c|c|c|c|}
\hline & \multirow{2}{*}{$\begin{array}{l}\text { Reference } \\
\text { values }\end{array}$} & \multicolumn{3}{|l|}{$\operatorname{HSMP}(n=27)$} & \multicolumn{3}{|l|}{$\operatorname{HSBP}(n=27)$} & \multirow[t]{2}{*}{$p$ value* } & \multirow[t]{2}{*}{$p$ value** } \\
\hline & & preoperative & postoperative & $p$ value & preoperative & postoperative & $p$ value & & \\
\hline \multicolumn{10}{|l|}{ INTEM } \\
\hline $\mathrm{CT}, \mathrm{s}$ & $100-240$ & $175(162-193)$ & $172(154-188)$ & 0.701 & $181(160-206)$ & $178(148-189)$ & 0.059 & 0.545 & 0.815 \\
\hline CFT, s & $30-110$ & $76(70-88)$ & $85(75-96)$ & 0.028 & $72(66-82)$ & $90(77-101)$ & $<0.001$ & 0.171 & 0.197 \\
\hline$\alpha\left(^{\circ}\right)$ & $70-83$ & $75(73-76)$ & $74(71-75)$ & 0.055 & $76(74-77)$ & $74(71-76)$ & 0.001 & 0.111 & 0.767 \\
\hline $\mathrm{MCF}, \mathrm{mm}$ & $50-72$ & $61(58-63)$ & $58(57-62)$ & 0.021 & $61(58-64)$ & $59(56-61)$ & 0.043 & 0.670 & 0.876 \\
\hline \multicolumn{10}{|l|}{ EXTEM } \\
\hline $\mathrm{CT}, \mathrm{s}$ & $38-79$ & $61(59-69)$ & $58(52-64)$ & 0.004 & $62(55-70)$ & $59(55-65)$ & 0.285 & 0.869 & 0.253 \\
\hline CFT, s & $34-159$ & $82(72-91)$ & $89(81-107)$ & 0.001 & $77(67-86)$ & $85(75-108)$ & 0.001 & 0.319 & 0.598 \\
\hline$\alpha\left(^{\circ}\right)$ & $63-83$ & $73(72-75)$ & $72(69-74)$ & 0.005 & $74(73-76)$ & $73(69-75)$ & 0.009 & 0.208 & 0.414 \\
\hline $\mathrm{MCF}, \mathrm{mm}$ & $50-72$ & $62(59-64)$ & $61(58-63)$ & 0.056 & $64(61-66)$ & $63(59-65)$ & 0.014 & 0.184 & 0.266 \\
\hline \multicolumn{10}{|l|}{ FIBTEM } \\
\hline $\mathrm{MCF}, \mathrm{mm}$ & $9-25$ & $12(10-14)$ & $11(10-12)$ & 0.001 & $14(10-17)$ & $12(10-15)$ & 0.005 & 0.143 & 0.206 \\
\hline
\end{tabular}

* $p$ value compare the preoperative values between the 2 groups.

** $p$ value compare the postoperative values between the 2 groups.

Data are expressed as median (interquartile range).

$\mathrm{p}$ value, compare the pre and postoperative values within groups.

ROTEM, rotational thromboelastometry; HSMP, monopolar hysteroscopic surgery group; HSBP, bipolar hysteroscopic surgery group; CT, clotting time; CFT, clot formation time; $\alpha$, alpha-angle; MCF, maximum clot firmness.

Table 3. Laboratory findings

\begin{tabular}{|c|c|c|c|c|c|c|c|c|}
\hline & \multicolumn{3}{|l|}{$\mathrm{HS}_{\mathrm{MP}}(n=27)$} & \multicolumn{3}{|l|}{$\mathrm{HS}_{\mathrm{BP}}(n=27)$} & $p$ value* & $p$ value ${ }^{* *}$ \\
\hline Hct, \% & $39.0(38.0-41.0)$ & $37.0(37.0-39.0)$ & $<0.001$ & $38.0(36.0-39.0)$ & $37.0(34.0-38.0)$ & $<0.001$ & 0.134 & 0.330 \\
\hline Platelet, $10^{9} / \mathrm{L}$ & $221(195-287)$ & $188(154-237)$ & $<0.001$ & $264(195-276)$ & $210.0(185-238)$ & $<0.001$ & 0.494 & 0.191 \\
\hline PT-INR & $1.01(1.00-1.07)$ & $1.09(1.01-1.10)$ & 0.148 & $1.01(1.00-1.05)$ & $1.03(0.98-1.11)$ & 0.364 & 0.807 & 0.573 \\
\hline $\mathrm{K}, \mathrm{mmol} / \mathrm{L}$ & $3.8(3.6-3.9)$ & $3.7(3.2-3.8)$ & 0.061 & $3.8(3.6-3.9)$ & $3.7(3.6-3.9)$ & 0.178 & 0.646 & 0.633 \\
\hline $\mathrm{Cl}, \mathrm{mmol} / \mathrm{L}$ & $107.5(106.9-109.6)$ & $107.3(106.8-109.0)$ & 0.780 & $107.3(106.6-109.1)$ & $107.9(106.6-109.5)$ & 0.946 & 0.677 & 0.472 \\
\hline
\end{tabular}

* $p$ value compare the preoperative values between the 2 groups.

** $p$ value compare the postoperative values between the 2 groups.

Data are expressed as median (interquartile range).

$p$ value, compare the pre and postoperative values within groups.

$\mathrm{Hb}$, hemoglobin; Hct, hematocrit; Na, sodium; K, potassium; Cl, chloride; PT-INR, international normalized ratio of prothrombin time; aPTT, activated partial thromboplastin time; HSMP, monopolar hysteroscopic surgery group; HSBP, bipolar hysteroscopic surgery group.

In the present study, we investigated and compared the changes in blood coagulation in accordance with 2 different irrigation solutions, an electrolyte-free hypotonic versus an isotonic solution. According to a previously published observational study [3], the absorption of nonelectrolyte solution during transurethral prostatectomy impaired the blood coagulation cascade, causing either an inhibition of the clotting factor activity or a de- crease in the coagulation factor concentration through hemodilution. Moreover, Istre et al. [12] reported that the coagulation parameters were indeed influenced by the amount of hypotonic solution absorbed. They showed that there was a greater change in the coagulation parameters with a greater volume of irrigation fluid absorption. Our results support these 2 studies with respect to coagulopathy. 
While hemodilution with isotonic saline has been reported to cause hypercoagulable change [13, 14], their findings differ from those of the current study in 2 ways: first, because their findings were obtained in an experimental environment, they may be of limited relevance to a clinical hemostatic system that is activated by hemodilution caused by irrigation fluid absorption or bleeding due to surgical tissue trauma. Second, the hemodilution level was $20 \%$ in those studies, indicating moderate hemodilution; however, in the present study, the median absorption amount was $200 \mathrm{~mL}$, which represents $<10 \%$ hemodilution. In spite of the mild hemodilution, hemostatic changes tended toward a hypocoagulable state.

According to our results, the median surgery time and irrigation fluid volume absorbed were $20-25 \mathrm{~min}$ and $200 \mathrm{~mL}$, respectively. Despite mild hemodilution with a short operation time, we observed a change in the coagulation cascade. Therefore, we suspect that with a prolonged surgery time and greater volumes of irrigation fluid absorption, which would ultimately increase hemodilution, greater shifts in the coagulation cascade would occur. As a result, in cases of prolonged surgery time, the coagulation state should be closely monitored. Most importantly, physicians should perform a scrupulous realtime evaluation of the balance between the volume of fluids irrigated and absorbed to prevent clinical intravasation syndrome during the surgery.

There were several limitations to consider in this study. First, this study only analyzed the coagulation changes in female patients with an ASA classification of 1 or 2 . Hence, the results may be subject to selection bias, thereby minimizing its generalizability to all populations. In general, patients with an ASA classification of $\geq 3$ may have underlying diseases, such as coronary artery or cerebrovascular diseases, requiring antiplatelet or antithrombotic medications. These drugs, despite discontinuation for several days before surgery, may still exert an influence on blood coagulation and the ROTEM analysis. Thus, we limited the condition of patients to those with an ASA classifica- tion of 1 or 2. Second, our results described coagulation changes with a relatively small absorption amount $(200 \mathrm{~mL})$. As such, the effects of moderate to severe dilution were not shown. Severe dilution is virtually impossible in a clinical setting as it poses severe risks to patients. To overcome this limitation, an in vitro study in which a profound hemodilution level can be induced may be valuable.

In conclusion, according to our ROTEM results, the volume of irrigation fluid absorbed significantly affects the blood coagulation cascade in healthy women undergoing HS, irrespective of the irrigant type. However, further studies are warranted to better clarify the relationship between the degree of absorption and degree of impact on coagulation and to determine the clinical implications of irrigation fluid absorption for patient safety.

\section{Statement of Ethics}

The study protocol has been approved by the research institute's committee on human research.

\section{Disclosure Statement}

The authors have no conflicts of interest to declare.

\section{Funding Source}

No external funding declared.

\section{Author Contributions}

H.-J.S.: Study design, Data collection, Manuscript writing, Final approval of the version to be submitted. H.-S.N.: Study design, Manuscript writing, Final approval of the version to be submitted. J.-Y.H.: Data analysis, Final approval of the version to be submitted. J.-W.H.: Manuscript writing, Final approval of the version to be submitted.

\section{References}

1 Hahn RG. Fluid absorption in endoscopic surgery. Br J Anaesth. 2006 Jan;96(1):8-20.

2 Munro MG. Complications of hysteroscopic and uterine resectoscopic surgery. Obstet Gynecol Clin North Am. 2010 Sep;37(3):399425.

3 Shin HJ, Na HS, Jeon YT, Park HP, Nam SW, Hwang JW. The impact of irrigating fluid absorption on blood coagulation in patients un- dergoing transurethral resection of the prostate: A prospective observational study using rotational thromboelastometry. Medicine (Baltimore). 2017 Jan;96(2):e5468.

4 Bergeron ME, Ouellet P, Bujold E, Cote M, Rhéaume $\mathrm{C}$, Lapointe $\mathrm{D}$, et al. The impact of anesthesia on glycine absorption in operative hysteroscopy: a randomized controlled trial. Anesth Analg. 2011 Oct;113(4):723-8.
5 Aydeniz B, Gruber IV, Schauf B, Kurek R, Meyer A, Wallwiener D. A multicenter survey of complications associated with 21,676 operative hysteroscopies. Eur J Obstet Gynecol Reprod Biol. 2002 Sep;104(2):160-4.

6 Cooper JM, Brady RM. Intraoperative and early postoperative complications of operative hysteroscopy. Obstet Gynecol Clin North Am. 2000 Jun;27(2):347-66.
Hemostasis and Irrigation Fluid in Hysteroscopic Surgery
Gynecol Obstet Invest 2019;84:568-574 DOI: $10.1159 / 000499914$ 
7 Goldenberg M, Zolti M, Seidman DS, Bider D, Mashiach S, Etchin A. Transient blood oxygen desaturation, hypercapnia, and coagulopathy after operative hysteroscopy with glycine used as the distending medium. Am J Obstet Gynecol. 1994 Jan;170(1 Pt 1): 25-9.

8 Schäfer M, Von Ungern-Sternberg BS, Wight E, Schneider MC. Isotonic fluid absorption during hysteroscopy resulting in severe hyperchloremic acidosis. Anesthesiology. 2005 Jul;103(1):203-4.

9 Rosencher N, Kerkkamp HE, Macheras G, Munuera LM, Menichella G, Barton DM, et al.; OSTHEO Investigation. Orthopedic Sur- gery Transfusion Hemoglobin European Overview (OSTHEO) study: blood management in elective knee and hip arthroplasty in Europe. Transfusion. 2003 Apr;43(4):45969.

10 Park JT, Lim HK, Kim SG, Um DJ. A comparison of the influence of $2.7 \%$ sorbitol- $0.54 \%$ mannitol and $5 \%$ glucose irrigating fluids on plasma serum physiology during hysteroscopic procedures. Korean J Anesthesiol. 2011 Nov;61(5):394-8.

11 Calabrese S, DE Alberti D, Garuti G. The use of bipolar technology in hysteroscopy. Minerva Ginecol. 2016 Apr;68(2):13342.
12 Istre O, Jellum E, Skajaa K, Forman A. Changes in amino acids, ammonium, and coagulation factors after transcervical resection of the endometrium with a glycine solution used for uterine irrigation. Am J Obstet Gynecol. 1995 Mar;172(3):939-45.

13 Ruttmann TG, James MF, Viljoen JF. Haemodilution induces a hypercoagulable state. $\mathrm{Br} \mathrm{J}$ Anaesth. 1996 Mar;76(3):412-4.

14 Roche AM, James MF, Bennett-Guerrero E, Mythen MG. A head-to-head comparison of the in vitro coagulation effects of saline-based and balanced electrolyte crystalloid and colloid intravenous fluids. Anesth Analg. 2006 Apr;102(4):1274-9. 